\title{
REVISIÓN
}

\section{Auras olfatorias en pacientes con epilepsia}

\author{
Niza Harari Masri,* Paul Shkurovich Bialik** \\ * Otorrinolaringología y Cirugía de Cabeza y Cuello. \\ ** Jefe del Departamento de Neurofisiología Clínica. Centro Neurológico. \\ Centro Médico ABC.
}

\begin{abstract}
RESUMEN
La epilepsia es una afección cerebral crónica caracterizada por crisis recurrentes, autolimitadas y de etiología muy variable, cuyas manifestaciones clínicas incluyen una gama de signos y síntomas en relación con la zona afectada. Las crisis uncinadas o alucinaciones olfatorias se describieron desde el siglo XIX como un olor particular, frecuentemente desagradable al inicio o durante la evolución de una crisis. La anatomía y propiedades únicas de la corteza olfatoria están involucradas en la epilepsia de lóbulo temporal, en donde hay integración de funciones sensitivas, incluyendo la olfatoria. El involucramiento de la corteza piriforme se asocia frecuentemente con la epilepsia de difícil control. En este artículo realizamos una revisión y análisis de la literatura actual sobre la anatomía y fisiología del olfato, así como su relación con la epilepsia.
\end{abstract}

Palabras clave: Epilepsia, crisis convulsivas, olfato, aura, corteza piriforme.

\begin{abstract}
Epilepsy is a chronic cerebral disease characterized by recurrent, self-limiting seizures of very variable etiology, whose clinical manifestations include a range of signs and symptoms related to the affected cortical zone. Uncinated seizures or olfactory hallucinations have been described since the 19 th century as a particular, often unpleasant smell at the beginning or during the spell. The anatomy and unique properties of the olfactory cortex are involved in temporal lobe epilepsy, where sensation modalities including smell are integrated. The involvement of the piriform cortex is associated with refractory epilepsy. In this article, we review and analyze the current literature on the anatomy and physiology of smell; and its relationship with epilepsy.
\end{abstract}

Keywords: Epilepsy, seizure, smell, aura, piriform cortex.

\section{INTRODUCCIÓN}

El sistema nervioso tiene la capacidad de interactuar con el medio ambiente por medio de una detección química, la cual ha evolucionado al sistema olfatorio principal, el sistema gustativo y el sistema trigeminal.

El olfato y el gusto son sentidos químicos que permiten decodificar la información por medio de moléculas volátiles como los olores e irritantes percibidos en la cavidad nasal, y moléculas no volátiles en la cavidad oral. A esto se agrega la sensación somatosensorial o trigeminal.

El olfato es considerado el más primitivo de los sentidos. El olfato se define como la capacidad de detectar, percibir, identificar y discriminar diferentes tipos de señales químicas (odorantes). ${ }^{1,2,3}$ El olfato es un sentido que se especializa en detectar moléculas volátiles, las cuales se vaporizan y alcanzan las fosas nasales, para ser absorbidas por la mucosa nasal e iniciar el proceso.
La percepción de los aromas resulta de la combinación de la activación del olfato por medio de la inhalación (ruta ortonasal) y la vía posterior (ruta retronasal).

El sentido del olfato influye en la conducta alimentaria e interacciones sociales, por lo que puede afectar la calidad de vida. Existe una gran conexión entre el olfato y la memoria, por lo que influye de forma importante en el estado emocional y toma de decisiones de la persona. ${ }^{1,4}$

En las últimas décadas, se han planteado numerosas teorías e hipótesis de cómo la función olfatoria puede ser indicador del inicio o curso de diversas enfermedades neurodegenerativas. ${ }^{1,3}$

La corteza piriforme es una región única del cerebro, la cual es responsable de los mecanismos de las experiencias olfatorias, forma la mayor parte de la corteza olfatoria primaria con extensas conexiones con distintas partes de la vía olfatoria. A diferencia de las regiones corticales primarias, recibe entrada 
directa del bulbo olfatorio sin tener paso por el tálamo. Además, es una estructura alocortical con tres capas, en el ser humano solamente se ha encontrado en el hipocampo, una de las regiones más implicadas en la epilepsia focal. ${ }^{4}$

El papel de la corteza piriforme no había sido ampliamente reconocido como parte de la epilepsia focal; esto fue en el siglo XIX, cuando se describieron las alucinaciones olfatorias llamadas "alucinaciones uncinadas", las cuales se creía que precedían a tumores del lóbulo temporal. Clínicamente se ha observado que pacientes con epilepsia tienen involucramiento de la corteza olfatoria.

En las últimas dos décadas en el campo de la epilepsia se han utilizado diferentes métodos y estudios como video-electroencefalografía, neuroimagen estructural y funcional, así como medicina nuclear para tratar de demostrar el involucramiento de la corteza piriforme. ${ }^{4,5}$

Se realiza una revisión bibliográfica de la anatomía y función de la vía olfatoria, asimismo de la corteza piriforme y su asociación con auras y crisis epilépticas.

\section{ANATOMÍA Y Fisiología DEL OLFATO}

Un olor se define como una impresión especial que deriva de una reacción volátil de algunas sustancias químicas sobre el sistema olfatorio. Diferentes elementos componen un olor, lo que le da características propias. ${ }^{1,2,3}$

Durante la inspiración, las moléculas odoríferas penetran la cavidad nasal, sólo el 10\% del aire penetra la hendidura olfatoria y la capacidad de detectar las moléculas dependerá de la velocidad del flujo de aire y la hidrosolubilidad de éstas. Las propiedades del transporte aéreo dependen de las características anatómicas nasales, aunque la cirugía funcional para mejorar la ventilación no siempre mejora el olfato. ${ }^{3}$

El neuroepitelio olfatorio es de tipo columnar pseudoestratificado, ${ }^{3}$ derivado embriológicamente de la placoda olfatoria y la cresta neural, ${ }^{1}$ es considerado el órgano receptor principal del olfato y se localiza en el techo de la cavidad nasal, predominantemente en la cara dorsal de la válvula nasal, septum y cornetes superiores. ${ }^{1,3,6}$ El límite anterior de la región olfatoria es 1-2 cm por delante de la inserción anterior del cornete medio.

La distribución exacta del neuroepitelio olfatorio no está entendida en su totalidad. ${ }^{6}$ Desde 1892, Von Brunn fue el primer médico en reportar las medidas de la región olfatoria reportando de $307-238 \mathrm{~mm}^{2}$ en cada fosa nasal; 50 años después, Smith reportó 134 $\mathrm{mm}^{2}$ e identificó cómo la región olfatoria disminuye a los 65 años en un 55\% y queda ausente hasta en un $13 \%$ en los adultos mayores. ${ }^{1-3,6-8}$ Un factor que hace que varíen estas medidas es la intermitencia entre el tejido olfatorio y el tejido respiratorio. En cada fosa nasal se encuentran de 10-20 millones de receptores olfatorios, ${ }^{1,9}$ éstos se producen de forma continua por mitosis y se pierden por descamación, con una duración del ciclo de tres meses aproximadamente. ${ }^{3}$

El neuroepitelio olfatorio está formado por neuronas olfatorias bipolares, las cuales actúan como receptores y transductores de forma simultánea. Además, posee glándulas olfatorias de Bowman, capaces de producir el líquido mucoso que disuelve las sustancias odoríferas. ${ }^{4}$

Las moléculas odoríferas activan receptores específicos localizados en los cilios de neuronas bipolares en la mucosa olfatoria, esto genera una respuesta que activa la proteína $\mathrm{G}$, la cual incrementa adenilato ciclasa 3 y esto, a su vez, AMP cíclico; esto promueve la entrada de calcio y la salida de cloro, logrando la despolarización de la célula. ${ }^{1}$ El ser humano cuenta con 350 receptores distintos y requiere la activación de 10 receptores para discriminar una molécula odorífera. ${ }^{2,3,7}$

Las células gliales olfatorias forman la vaina de las neuronas olfatorias primarias. Los axones se agrupan en 20 fascículos aproximadamente, que luego formarán el nervio olfatorio, primer nervio craneal, el cual atraviesa la lámina cribiforme del etmoides para entrar a la cavidad craneal, y se continúa hacia el bulbo olfatorio formando glomérulos. ${ }^{2,3}$

Los axones de las células mitrales se proyectan de forma ipsolateral a la corteza olfatoria primaria y a través del trayecto olfatorio lateral. Las células principales del bulbo olfatorio terminan en áreas olfatorias primarias: núcleo olfatorio anterior, corteza piriforme, núcleo amigdaloide cortical anterior, corteza periamigdalina y corteza entorrinal; ;,4,10 además de estas regiones, la red olfatoria involucra la corteza orbitofrontal, tálamo e ínsula ${ }^{11}$ para la apreciación subjetiva de los olores, y posteriormente establecer conexiones con otras áreas encefálicas para respuestas emocionales y viscerales.

El área olfatoria, llamada corteza piriforme presente en el lóbulo temporal, es considerada filogenéticamente más antigua que la neocorteza y cuenta con un centro especializado para el sentido del olfato. ${ }^{12}$

La corteza piriforme tiene un rol importante en la percepción y discriminación de los olores y actúa como un punto de distribución espacial importante, 
el cual no tiene una actividad estática y muestra cambios según la fase del ciclo respiratorio. ${ }^{4,13}$ La porción anterior de la corteza piriforme es la encargada de decodificar los olores, y la posterior, de la calidad de los mismos. Existe una habituación rápida de los olores a la respuesta de la corteza piriforme; ${ }^{3}$ la activación del sistema límbico para procesar el estímulo de un olor juega un papel importante en la memoria, emociones y comportamiento. Durante la identificación de un olor, la corteza entorrinal e hipocampo se activan e inicia la reactivación de la memoria. ${ }^{14}$

Las sensaciones somatosensoriales de la mucosa nasal son mediadas por el sistema trigeminal, el cual contribuye de forma indirecta en la percepción de los olores. La cavidad nasal está inervada por la rama oftálmica y maxilar del nervio trigeminal, las ramas del nervio oftálmico (nervio etmoidal anterior e infraorbitario) inervan la porción anterior de la cavidad nasal. La inervación de la porción posterior de la cavidad nasal es dada por fibras de la rama maxilar (nervio nasal medial superoposterior y nasopalatino). Por estudios electrofisiológicos se demuestra que el tercio anterior de la cavidad nasal tiene mayor actividad trigeminal mediante quimiorreceptores. ${ }^{15}$

Mediante diversas estructuras receptoras, las sensaciones son mediadas por la vía aferente trigeminal (tacto, temperatura y nocicepción); la estimulación química es importante ya que la mucosa no se encuentra cubierta por epitelio escamoso, dando un estímulo químico casi directo a los nervios y activando diversos receptores. Los cuerpos celulares aferentes trigeminales se localizan en el ganglio de Gasser, sus axones se proyectan al subnúcleo sensorial trigeminal, núcleos espinales y mesencefálicos del trigémino que se extienden de la médula espinal rostral al mesencéfalo, éstos descienden por el trayecto trigeminal y terminan en el núcleo espinal, para que la información trigeminal sea transmitida a la amígdala por la vía parabranquial lateral. Las neuronas del núcleo espinal se proyectan hacia el tálamo y la mayoría de las fibras ascendentes lo hacen de forma contralateral, aunque algunas lo hacen de forma ipsolateral. Desde el tálamo las fibras se proyectan a la corteza somatosensorial primaria, además se demuestra actividad en la corteza insular y corteza orbital ventral. ${ }^{15}$

Se ha demostrado que el sistema trigeminal es menos sensible que el sistema olfatorio, la mayoría de los estímulos quimiosensoriales activan el sistema trigeminal y el olfatorio, por lo que es difícil identificar su activación de forma separada. Ambos sistemas aportan contribuciones separadas para poder percibir la intensidad de un estímulo complejo, ${ }^{15}$ algunos estimulantes producen efecto exclusivamente sobre el sistema olfatorio o sensaciones trigeminales, pero la mayoría estimulan las dos vías de forma simultánea, olor e irritación. En una revisión de 47 olores distintos se reveló que sólo dos estímulos por vainilla y ácido cúprico no tuvieron ninguna respuesta trigeminal en pacientes con anosmia, a diferencia del dióxido de carbono, que activó de forma selectiva el sistema trigeminal. ${ }^{15}$ La función primaria del sistema trigeminal es actuar como centinela de la vía aérea para limitar la respiración y prevenir la inhalación de sustancias potencialmente peligrosas.

Existen diversas enfermedades neurodegenerativas en donde se ve afectado el sentido del olfato, y éste puede ser el indicador del inicio o curso de las mismas.

\section{EPILEPSIA}

La Comisión de Clasificación y Terminología de la ILAE (Liga Internacional Contra la Epilepsia) define una crisis epiléptica como la aparición transitoria de signos y/o síntomas debido a una actividad neuronal excesiva o sincrónica en el cerebro. El aura epiléptica corresponde a una crisis de inicio focal y se describe como el fenómeno ictal subjetivo que puede preceder a una crisis tangible, para la cual se mantiene la consciencia; ${ }^{16,17}$ fisiopatológicamente es el resultado de la activación de un área cortical funcional por una descarga neuronal anormal, unilateral, localizada y breve, pueden ocurrir de forma aislada o progresar hacia una crisis parcial compleja con afección de la consciencia o hacia una crisis tónico-clónica generalizada. Usualmente son el primer síntoma en presentarse en el curso de una crisis, por lo que son el mejor indicador clínico para la posible localización del foco epiléptico, mas no la lateralización de la patología. ${ }^{18,19}$

La duración de las auras es variable, desde segundos hasta varios minutos (Figura 1). Se realizó un estudio utilizando monitoreo video-electroencefalográfico con electrodos extracraneales estableciendo una duración aproximada de 87 segundos de auras motoras y 63 segundos en sensitivas.

La corteza piriforme es una región única del cerebro, con una estructura filogenéticamente antigua, forma la mayor parte de la corteza olfatoria primaria y tiene una estructura alocortical, al igual que el hipocampo. ${ }^{4}$ Históricamente, el papel de la corteza piriforme en la epilepsia no estaba ampliamente reconocido o estudiado, se prestó la mayor atención a la región temporal mesial, en especial al hipocampo. 
En el siglo XIX, se describieron las auras olfatorias por Hughlings Jackson y Stewart, 17,20 llamadas "crisis uncinadas"; las cuales se describían como sensaciones desagradables que percibían los pacientes. ${ }^{1,3,21}$ Ferrier realizó estudios en animales, con los cuales concluyó que el olfato y gusto se localizan en el giro uncinado. Previamente a esto Sander, en 1874, describió pacientes en los cuales, antes de sus crisis, percibían un "olor desagradable", seguido de movimientos masticatorios y salivación. Las auras olfatorias pueden acompañarse de auras emocionales en pacientes con esclerosis de lóbulo temporal, y se acompaña de sensación epigástrica y automatismos. ${ }^{20}$ Cuando existen diferentes tipos de auras en la misma crisis, el orden de la progresión indica la dirección de la crisis.

En 1980, se descubrió que la corteza piriforme era un tejido epileptógeno, esto en modelos animales, sin ningún impacto clínico en seres humanos. En las últimas dos

A

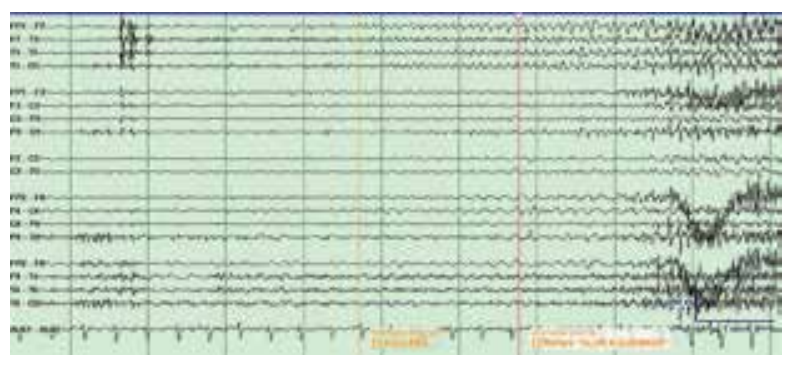

B

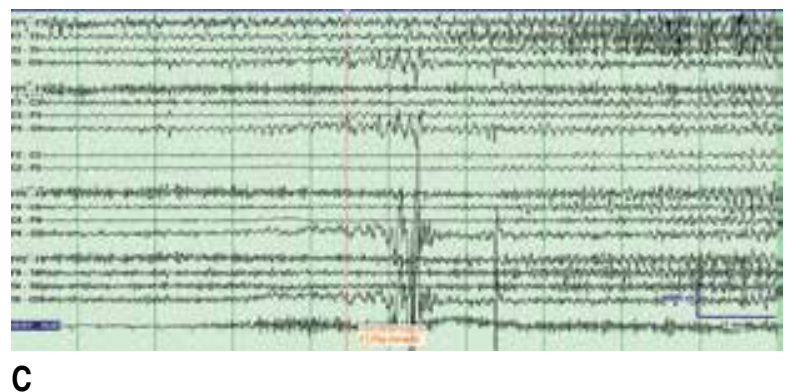

C

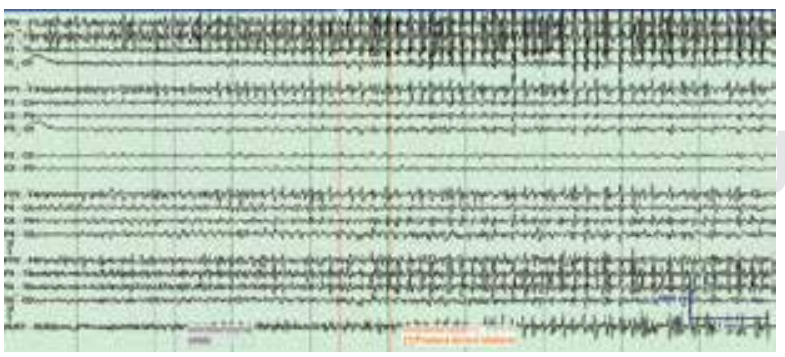

Figura 1. Épocas de una paciente con crisis uncinadas, al inicio del evento y durante la evolución electroencefalográfica. décadas se han realizado estudios de imagen, fisiológicos y funcionales que demuestran cómo la corteza piriforme puede ser un punto crucial en la epilepsia focal. ${ }^{4}$

Las auras olfatorias generalmente son descritas como desagradables, se ha reportado una prevalencia en la literatura en un rango de 0.6 a $16 \%$ en pacientes con epilepsia de lóbulo temporal ${ }^{1,3,16}$ y se asocian con tumores en estructuras temporomesiales y orbitofrontales. ${ }^{16}$ En un estudio reciente de Acharya y colaboradores, se reportó un $0.9 \%$ de auras olfatorias en pacientes con epilepsia de difícil control, ${ }^{16,20,22}$ seleccionaron 1,432 pacientes con epilepsia parcial sin respuesta a fármacos y analizaron sus características clínicas, video-encefalográficas y neuroimagen; en 13 pacientes se reportaron auras olfatorias y en siete de ellos sensaciones no placenteras, en todos ellos precedió una crisis parcial compleja y en 10 pacientes se detectó un tumor en lóbulo temporal mesial con afección de la amígdala e hipocampo. ${ }^{23}$

Debido al acomodo y gran cantidad de ramificaciones entre las células piramidales en la corteza piriforme, se forma una red neuronal excitatoria extensa, la cual requiere una potente inhibición para prevenir la propagación descontrolada de la actividad eléctrica, por lo que se considera un área altamente

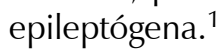

La vía olfatoria y el sistema límbico comparten estructuras involucradas en la epilepsia temporal mesial, la interacción entre la circunvalación frontal inferior dominante, la circunvalación fusiforme y la región temporal; la conexión entre ellas se vuelve susceptible al daño excitatorio de la actividad eléctrica descontrolada. $^{1}$

La sensibilidad olfativa en pacientes epilépticos es variable, algunos estudios reportan un umbral olfatorio normal, mientras que otros reportaron anormalidades en tareas olfatorias específicas como discriminación, reconocimiento de olores a corto y largo plazo y dificultad para identificar o nombrar algún olor. ${ }^{1,21,24} \mathrm{Sin}$ embargo, debido a las diferentes variables utilizadas en los múltiples estudios, ha sido imposible determinar algún patrón de deficiencia específico, así como la localización y lateralidad del foco epiléptico o la duración y frecuencia de las crisis. Se han realizado estudios utilizando resonancia magnética funcional y colocación de electrodos profundos en hipocampo, lo que demostró una disfunción con poca o nula bilateralidad de áreas del sistema olfatorio, por lo cual ha sido imposible correlacionarla con la lateralidad del foco epiléptico. 
En pacientes con epilepsia focal hay una superposición de las regiones epileptógenas y las regiones de la red sensorial que produce alteraciones. Pacientes con epilepsia de lóbulo temporal presentan múltiples alteraciones de la función olfatoria; ${ }^{25}$ el umbral de detección a diversos olores como butano o alcohol fenetílico es normal en estos pacientes, aunque utilizando diversos paneles de olores, los umbrales a la detección de los olores están alterados, siendo más sensibles en el pródromo de la crisis y reduciendo la sensibilidad en la fase postictal. ${ }^{24,25}$ En contraste a esto, la discriminación de los olores se da en la corteza piriforme, región orbitofrontal e hipocampo, y la falla en esta función refleja la disfunción de esta red, ${ }^{26-29}$ en varios estudios se ha demostrado daño en la memoria del olfato. ${ }^{30}$

Múltiples modalidades de neuroimagen demuestran cambios en la corteza piriforme en pacientes con epilepsia focal, paralelos a la disfunción del procesamiento olfatorio. Con resonancia magnética volumétrica se demuestra atrofia en la corteza piriforme del lóbulo temporal, identificando reducción del volumen en el mismo lado que el foco epiléptico. ${ }^{25}$

Estudios de potenciales quimiosensoriales nos hablan del tiempo relativo del procesamiento olfativo, en pacientes con epilepsia de lóbulo temporal estos potenciales están retrasados del lado del foco epiléptico. Este efecto es más pronunciado en epilepsia de lóbulo temporal derecho, reflejando mayor importancia del olfato en dicho hemisferio. ${ }^{31}$

La actividad funcional del olfato de las regiones del cerebro se ha estudiado con tomografía con emisión de positrones, en pacientes con epilepsia de lóbulo temporal se ha encontrado hipoactividad en la corteza piriforme ipsolateral, amígdala e ínsula anterior con diferentes olores.

Múltiples estudios han concluido que la epilepsia temporal mesial con esclerosis en hipocampo demostrada por resonancia magnética tiende a ser más resistente al control con fármacos antiepilépticos. Estos pacientes presentan auras de forma aislada, las auras olfatorias se presentan en menos de $5 \%$ de los pacientes. Un estudio realizado por Fried y colaboradores describe que las auras epigástricas, gustativas y olfatorias son significativamente más frecuentes en pacientes con epilepsia temporal mesial con esclerosis en hipocampo ${ }^{23}$ que en pacientes con lesiones temporales neocorticales o extratemporales. Existen ocasiones en que, después de cirugías exitosas, los pacientes quedan libres de crisis epilépticas, pero continúan presentando auras. De los pacientes con esclerosis en hipocampo posterior a la resección quirúrgica, $18.9 \%$ persistió con las auras epilépticas a diferencia del $2.6 \%$ con lesiones temporales neocorticales o extratemporales. ${ }^{18}$

\section{DiSCUSIÓN Y CONCLUSIONES}

El sentido del olfato cambia con el tiempo por un proceso de envejecimiento normal, afectando las habilidades de detectar, identificar y discriminar olores. Varias enfermedades neurodegenerativas presentan ciertas alteraciones que nos ayudan a determinar su origen y progresión.

Un aura es un fenómeno subjetivo que precede a la crisis convulsiva, signo clínico que puede presentarse previo a la pérdida del estado de consciencia.

Las manifestaciones clínicas de las auras epilépticas son extraordinariamente diversas, el conocimiento adecuado de su fisiopatología, características, incidencia y asociación con los distintos tipos de epilepsia focal es importante para poder determinar el posible sitio de localización del foco epiléptico.

Las auras olfatorias se consideran episodios que se desencadenan por una crisis epiléptica del lóbulo temporal, en particular de la región mesial con estimulación simultánea de estructuras corticales, observando una fuerte relación con la amígdala, corteza entorrinal e ínsula rostral, los cuales tienen la mayor cantidad de proyecciones hacia el bulbo olfatorio.

La evidencia disponible nos enseña que los estímulos químicos o eléctricos repetidos aplicados en regiones límbicas pueden producir cambios complejos en la corteza piriforme, resultando en aumento de la excitabilidad y ésta proporcionar una vía para la distribución de descargas epilépticas. ${ }^{4} \mathrm{El}$ rol de la corteza piriforme durante la epileptogenis es probable, pero continúa sin ser confirmado.

Se han realizado múltiples estudios donde los resultados son consistentes en que existen anormalidades en las funciones del olfato, discriminación, tiempo de reconocimiento de un olor y dificultad para nombrar un olor; sin embargo, las diferentes metodologías utilizadas no han permitido definir un patrón de lateralización del foco epiléptico, la duración o frecuencia de las crisis.

Se infiere que las crisis parciales que inician con una sensación olfatoria se propagan desde la corteza piriforme. Los fenómenos olfatorios más frecuentes son las alucinaciones, donde la percepción del olor no está relacionada con algún estímulo del medio ambiente. También existen las ilusiones olfatorias, en las cuales un olor del medio ambiente es percibido de manera incorrecta. 
Debemos considerar que la corteza piriforme tiene influencia en la progresión a una epilepsia de difícil control, definida clínicamente cuando existen crisis epilépticas continuas, a pesar de una prueba terapéutica adecuada con dos fármacos antiepilépticos bien tolerados y dosificados.

Por lo tanto, la corteza piriforme debe ser considerada de suma importancia en la epilepsia focal, la cual se origina en el lóbulo temporal, dado el probable involucramiento en la distribución de la descarga epiléptica; por esto podría ser considerado un objetivo en tratamientos quirúrgicos.

La patología del olfato es poco conocida y subdiagnosticada, pero puede resultar muy incapacitante, los síntomas no deben considerarse como menores, ya que tienen un impacto en la calidad de vida del paciente. Se debe tener en cuenta que el abordaje se debe realizar de forma multidisciplinaria, con énfasis en una importante historia clínica.

\section{Bibliografía}

1. Aguilar M, Aguado G, Saucedo PE, Mendoza CA, Velasco $\mathrm{AL}$, Velasco $\mathrm{F}$. Clinical importance of olfactory function in neurodegenerative diseases. Rev Med Hosp Gen Méx. 2018; 81 (4): 268-275.

2. Fuentes A, Javiera M, Santander H, Valenzuela S, Gutiérrez MF, Miralles R. Sensopercepción olfatoria: una revisión. Rev Med Chile. 2011; 139: 362-367.

3. Rennaker RL, Chen CFF, Ruyle AM, Sloan AM, Wilson DA. Spatial and temporal distribution of odorant-evoked activity in the piriform cortex. J Neurosci. 2007; 27: 1534-1542.

4. Vaughan DN, Jackson GD. The piriform cortex and the human focal epilepsy. Frontier in Neurology. 2014; 5: 259.

5. Carrillo B, Carrillo V, Astorga A, Hormachea D. Diagnóstico en la patología del olfato: Revisión de la literatura. Rev Otorrinolaringol Cir Cabeza Cuello. 2017; 77: 351-360.

6. Leopold D, Hummel T, Schwob J, Chen Hong S, Knecht M, Kobal G. Anterior distribution of human olfactory epithelium. Laryngoscope. 2000; 110: 417-421.

7. Landis BN, Simona N, Friedrich H. Chemosensory event related potential. Epileptologie. 2016; 33: 189-196.

8. Doty RL, Kamath $\mathrm{V}$. The influences of age in olfaction: a review. Front Psychol. 2014; 5: 1-20.

9. Soudry Y, Lemogne C, Malinvaud D, Consoli SM, Bonfils P. Olfactory system and emotion: common substrates. European Annal of Otorhinolaryngology, Head and Neck Diseases. 2011; 128: 18-23.

10. Carmichael ST, Clugnet MC, Price JL. Central olfactory connections in the macaque monkey. J Comp Neurol. 1994; 346: 403-434.

11. Shipley M, Reyes P. Anatomy of the human olfactory bulb and central olfactory pathways. The Human Sense of Smell. 1991; 29-60.

12. Mai JK, Paxinos G, Voss T. Atlas of the human brain. Amsterdam: Elsevier; 2008.

13. Mainland J. The sniff is part of the olfactory percept. Chem Senses. 2005; 31: 181-196.
14. Kjelvik G, Evensmoen HR, Brezova V, Håberg AK. The human brain representation of odor identification. J Neurophysiol. 2012; 108: 645-657.

15. Hummel T, Livermore A. Intranasal chemosensory function of trigeminal nerve and aspects of its relation to olfaction. Int Arch Occup Environ Health. 2001; 75: 305-313.

16. Fernández-Torre JL. Auras epilépticas: clasificación, fisiopatología, utilidad práctica, diagnóstico diferencial y controversias. Rev Neurol. 2003; 34 (10): 977-983.

17. Palacios E, Clavijo-Prado C. Semiología de la crisis epiléptica: un reto clínico. Repert Med Cir. 2016; 25 (4): 203-209.

18. Fried I, Spenser D, Spenser S. The anatomy of epileptic auras: focal pathology and surgical outcome. J Neurosurg. 1995; 83: 60-66.

19. Ferrari-Marinho T, Caboclo L, Marinho M, Centeno R, Neves $R$, Santana $T$ et al. Auras in temporal lobe epilepsy with hippocampal sclerosis: relation to seizure focus laterality and post surgical outcome. Epilepsy \& Behavior. 2012; 24: 120-125.

20. Chen C, Shih Y, Yen D, Lirng J, Guo Y, Yu H et al. Olfactory auras in patients with temporal lobe epilepsy. Epilepsia. 2003; 44 (2): 257-260.

21. Liu Y, Guo X, Wu X, Li P, Wang W. clinical analysis of partial epilepsy with auras. Chinese Medical Journal. 2017; 130 (3): 318-322.

22. Acharya V, Acharya J, Luders H. Olfactory epileptic auras. Neurology. 1998; 51 (1): 56-61.

23. Wieser HG. ILAE Commission report mesial temporal lobe epilepsy with hipocampal sclerosis. Epilepsia. 2004; 45 (6): 695-714.

24. McIntyre D, Gilby K. Mapping seizure pathways in the temporal lobe. Epilepsia. 2008; 49 (3): 23-30.

25. West SE, Doty RL. Influence of epilepsy and temporal lobe resection on olfactory function. Epilepsia. 1995; 36: 531-542.

26. Martinez BA, Cain WS, deWijk RA, Spencer DD, Novelly RA, Sass KJ. Olfactory functioning before and after temporal lobe resection for intractable seizures. Neuropsychology. 1993; 7: 351-363.

27. Savic I, Bookheimer SY, Fried I, Engel J Jr. Olfactory bed side test. A simple approach to identify temporo-orbitofrontal dysfunction. Arch Neurol. 1997; 54: 162-168.

28. Hudry J. Olfactory short-term memory and related amygdala recordings in patients with temporal lobe epilepsy. Brain. 2003; 126: 1851-63.

29. West SE, Doty RL, O'Connor MJ, Sperling MR. Pre and postoperative studies of olfactory function in patients with anterior temporal lobectomy. In: Proceedings of the Fifteenth Annual Meeting of the Association for Chemoreception Sciences, Sarasota, Florida. Chem Senses. 1993; 21: 649.

30. Hummel T, Henkel S, Negoias S, Galván JRB, Bogdanov V, Hopp $\mathrm{P}$ et al. Olfactory bulb volume in patients with temporal lobe epilepsy. J Neurol. 2013; 260: 1004-1008.

31. Hummel T, Pauli E, Schüler P, Kettenmann B, Stefan H, Kobal G. Chemosensory event related potentials in patients with temporal lobe epilepsy. Epilepsia. 1995; 36: 79-85.

\section{Correspondencia:}

\section{Dr. Niza Harari Masri}

Antiguo Camino a Tecamachalco Núm. 685-601,

Col. Lomas del Olivo, 52788,

Huixquilucan, Estado de México.

Teléfono casa: 62786643

E-mail: nizaharari@gmail.com 